\title{
Phenotypic switching of Cryptococcus neoformans occurs in vivo and influences the outcome of infection

\author{
Bettina C. Fries, ${ }^{1}$ Carlos P. Taborda, ${ }^{2}$ Evan Serfass, ${ }^{2}$ and Arturo Casadevall ${ }^{1,2}$ \\ ${ }^{1}$ Department of Medicine, and \\ ${ }^{2}$ Departments of Microbiology and Immunology, Albert Einstein College of Medicine, Bronx, New York, USA \\ Address correspondence to: Bettina Fries, Department of Medicine, Albert Einstein College of Medicine, \\ Golding 702, 1300 Morris Park Avenue, Bronx, New York 10461, USA. \\ Phone: (718) 430-3768; Fax: (718) 430-8701; E-mail: fries@aecom.yu.edu.
}

Received for publication May 31, 2001, and accepted in revised form October 9, 2001.

Phenotypic switching has been linked to the virulence of many pathogens, including fungi. However, it has not been conclusively shown to occur in vivo or to influence the outcome of infection. Cryptococcus neoformans undergoes phenotypic switching in vitro to colony types that differ in their virulence in mice. In this study, we asked whether C. neoformans undergoes phenotypic switching in vivo and whether this phenomenon contributes to virulence. By using a small inoculum to preclude the introduction of variants that had already switched during in vitro propagation, we demonstrated that in vivo switching to a mucoid phenotype occurred in two mice strains and was associated with a lethal outcome. Phenotypic switching resulted in changes of the capsular polysaccharide that inhibited phagocytosis by alveolar macrophages. This promoted a more vigorous inflammatory response and rapid demise. These data document in vivo switching in a fungus and associate this phenomenon with enhanced virulence and a lethal outcome. The importance of this finding is underscored by the increased likelihood of phenotypic switching in chronic cryptococcosis; thus this mechanism may account for the inability to eradicate the organism in immunocompromised hosts.

J. Clin. Invest. 108:1639-1648 (2001). DOI:10.1172/JCI200113407.

\section{Introduction}

Cryptococcosis affects $5-30 \%$ of patients with AIDS. It is caused by Cryptococcus neoformans, an encapsulated yeast that is acquired by inhalation and ultimately results in a chronic meningoencephalitis with a high tendency to relapse despite effective antifungal therapy (1). Several virulence factors (2-5) including a polysaccharide capsule $(6,7)$ are described for $C$. neoformans. Most virulence factors are invariably present (8) in clinical strains. Genetic inactivation of their regulatory proteins, which are part of signaling pathways, renders the yeast avirulent $(9,10)$. It is, however, unclear whether regulation of virulence factors occurs in vivo during chronic infection in order to enhance the virulence of an infecting C. neoformans strain. Therefore, it is often assumed that failure to eradicate the fungus is a function of a diminished cellmediated immune response (CMI). Several observations, however, indicate that pathogen-specific factors contribute to the pathogenesis of cryptococcosis. First, prolonged in vitro and in vivo passage of C. neoformans isolates can result in phenotypic changes associated with differences in virulence (11-14). Second, serial isolates from chronically infected patients can exhibit differences in virulence (15). Third, C. neoformans variety gattii strains cause symptomatic infections primarily in immunocompetent hosts even in areas where AIDS is endemic (16).
Reversible phenotypic switching can result in microevolution, defined as rapid changing of an organism to a stable, inherited phenotype (17). Microevolution and phenotypic switching can be achieved by several mechanisms, some of which involve changes of genes and follow a mendelian inheritance pattern whereas others (e.g., silencing) are epigenetic and inherited in a nonmendelian fashion. For certain pathogens, the emergence of phenotype variants is associated with invasion of mucosal barriers and immune evasion (18). For fungi, phenotypic switching is an in vitro phenomenon defined as the spontaneous emergence of colonies with altered colony morphology at rates higher than the somatic mutation rates (19-21). Phenotypic switching was demonstrated for C. neoformans in vitro in three different clinical strains and resulted in enhanced virulence $(22,23)$. Despite an association with virulence, phenotypic switching in vivo has been demonstrated only in pathogens with complex life cycles that require the switch for survival in the host (24). Most infections require high doses, and because of the expected in vitro switching rates $\left(10^{-2}\right.$ to $\left.10^{-5}\right)$, these inocula are likely to be contaminated with in vitro switch variants. Therefore, it is difficult to demonstrate that the emergence of new phenotypes in the infected host are the result of in vivo switching events and do not represent in vivo selection of variants from an initially heterogeneous pathogen population (25). 
Our objective was to investigate whether phenotypic switching of C. neoformans occurred in vivo and, if so, to explore its consequences. Our data show that phenotypic switching occurs in vivo and results in changes of a key virulence factor, the capsular polysaccharide. These findings suggest a unique mechanism of increased virulence, namely, an enhanced inflammatory response with altered cytokine and chemokine expression that ultimately interferes with the host ability to mount an effective immune response.

\section{Methods}

C. neoformans strain. Isolate RC-2 is a variant of serotype D strain 24067 (13) from the American Type Culture Collection (ATCC, Rockville, Maryland, USA). Melanization, cell charge, capsule and cell size, sugar assimilation profile, karyotype, and RFLP typing were determined as previously described $(13,26,27)$. The strain was streaked to single colonies and maintained on Sabouraud Dextrose Agar (SDA). RC-2 switches between a smooth (SM) and mucoid (MC) colony type, which are both common colony morphologies in clinical isolates. Switching was determined by visually scoring the colonies derived from homogenized organs after growth on SDA. For calculation of switching frequencies, approximately $10^{4}$ colonies were scored and the percentage of MC colonies of the total CFU was determined.

Characterization of SM and MC glucuronoxylomannan. Glucuronoxylomannan (GXM), the major capsular polysaccharide of C. neoformans, was purified from supernatants and analyzed by nuclear magnetic resonance (NMR) (11) and for elemental composition. The relative molecular weight was measured by size exclusion chromatography and zonal fraction ultracentrifugation. For size exclusion chromatography, a Sephacryl column (S-1000, $1 \times 39$ cm; Amersham Pharmacia Biotech, Piscataway, New Jersey, USA) was used and molecules (1-10 million daltons [mDa]) were eluted with PBS. For ultracentrifugation, GXM was dissolved in $1 \mathrm{ml}$ of PBS, layered on $11 \mathrm{ml}$ of a $5-20 \%$ sucrose gradient, and spun at $150,000 \mathrm{~g}$ in an SW 28.1 rotor (Beckman Instruments Inc., Fullerton, California, USA) for 12 hours at $20^{\circ} \mathrm{C}$. Fractions $(18 \times 0.75 \mathrm{ml})$ were collected from the bottom of the tube using a 14-gauge needle. GXM content in fractions was measured by the phenol sulfuric acid (chromatography) or capture ELISA (ultracentrifugation). Viscosity of the GXM dissolved in $\mathrm{H}_{2} \mathrm{O}$ was measured using a capillary viscometer (Technical Glass Products Inc., Dover, New Jersey, USA).

Animal studies. BALB/c and A/JCr mice (male, 6-12 weeks old) were obtained from the National Cancer Institute (Bethesda, Maryland, USA). Anesthetized mice were infected by inoculation of $10^{3}-10^{6} \mathrm{C}$. neoformans cells in $50 \mu \mathrm{l}$ sterile PBS into the trachea using a 26-gauge needle. When virulence of SM and MC was compared, dilutions of the inoculum were plated onto SDA to assure that comparable numbers of yeast cells were injected. Mice were observed daily for signs of disease. Percentage of MC colonies and CFU were determined by homogenizing lung and brain tissue in PBS, and plating dilutions of the homogenate on SDA. All protocols were done according to guidelines provided by the Albert-Einstein College of Medicine's animal use and protection committee.

Phagocytosis assays. In vitro phagocytosis assays were performed with murine macrophage $(\mathrm{M} \phi)$ cell lines (J774.16 and MH-S from ATCC) and primary peritoneal and alveolar M $\phi$ 's. Phagocytosis assays were performed using formaldehyde or heat-killed $\left(50^{\circ} \mathrm{C}\right) \mathrm{C}$. neoformans cells in the presence of $10 \mu \mathrm{g} / \mathrm{ml}$ of purified $\mathrm{IgG}_{1} \mathrm{mAb}$ to GXM (18B7 or $\left.2 \mathrm{H} 1\right)$. C. neoformans cells were added at a ratio of 2:1 for J774 cells, and 1:5 for primary M $\phi$ 's and MH-S cells. After 2 hours, monolayers were washed, fixed, and stained, and the phagocytosis index (PI) was determined (28). Experiments were performed in triplicate and repeated.

\section{Equation 1}

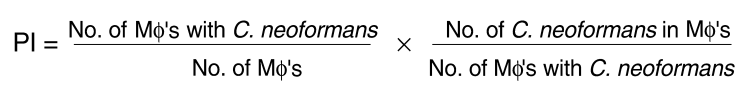

For in vivo phagocytosis assays, groups of BALB/c mice $(n=3)$ were infected with $10^{6} \mathrm{SM}$ or MC cells. After 4 hours, mice were killed and underwent bronchial lavage. Alveolar M $\phi$ 's were allowed to adhere for 1 hour at $37^{\circ} \mathrm{C}$, then fixed and stained to determine PI. In vitro killing assays were performed to assess intracellular survival of fungal cells. SM and MC cells were added at a $1: 10$ ratio to $\mathrm{MH}-\mathrm{S}$ cells $\left(4 \times 10^{4}\right.$ cells per well $)$ with $\mathrm{mAb}$ $18 \mathrm{~B} 7$, and incubated at $37^{\circ} \mathrm{C}$ for 2 hours. The initial PI was determined after washing the layers with PBS to remove nonadherent fungal cells. The ability of SM and MC to replicate in MH-S cells was determined by counting the CFU after coculture in the presence (intracellular replication) and absence of $\mathrm{mAb} 18 \mathrm{~B} 7$ (control for replication in media). Cells were lysed after 2, 6, 12, 24, 36 , and 48 hours of incubation. Supernatants and lysates were plated on SDA to determine CFU. Because the PI of SM is bigger than that of MC, intracellular replication was corrected for the initial PI (2 hours).

Histology and immunobistochemistry. Anesthetized mice were perfused with $4 \%$ paraformaldehyde in PBS for 3 minutes at $200 \mathrm{mmHg}$ followed by $18 \%$ sucrose in PBS for 5 minutes for fixation. Tissue sections were stained with hematoxylin and eosin (H\&E) or mucicarmine. Immunohistochemistry staining was done with an M $\phi$-specific $\mathrm{mAb}$ (Mac 3; Pharmingen, San Diego, California, USA) as primary antibody. Antigen detection was enhanced by microwave radiation for 30 minutes prior to staining. A biotinylated goat anti-rat IgG was used for detection and developed by incubation in diaminobenzidine.

Lung leukocyte isolation and FACS analysis of leukocyte subsets. Single cell suspensions were obtained by mincing, and enzymatically digesting excised lungs of infected mice as described (29). Erythrocytes were lysed and cells were counted in a hemocytometer. Subsets of isolated leukocytes were determined by Wright-Giemsa staining of samples cytospun onto slides, and by FACS analysis. 
a

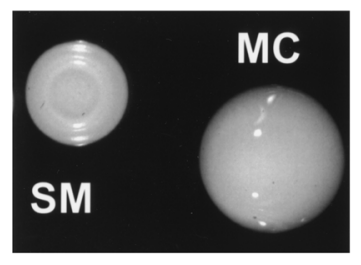

b

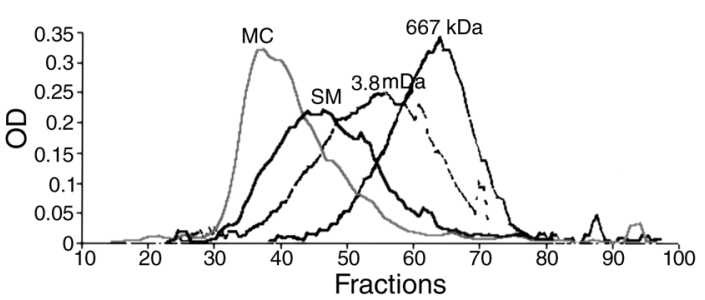

c

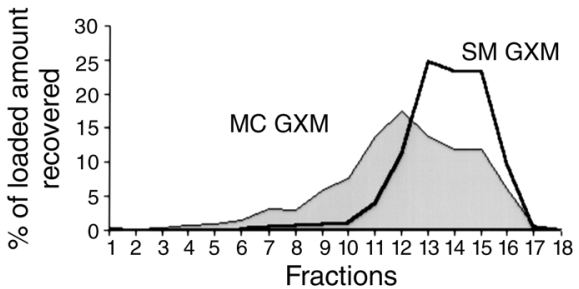

Figure 1

Characterization of SM and MC. (a) Mucoid colony surface of MC colony compared with the SM colony after growth on SDA for 72 hours. (b) Size exclusion chromatography shows earlier elution of MC GXM compared with SM GXM. (c) Zonal fractionation confirms larger size of MC GXM, which is detected in the earlier fractions after ultracentrifugation over a $5-20 \%$ sucrose gradient. mDa, million dalton.

For FACS analysis, cells were incubated for 30 minutes on ice with $0.20 \mu \mathrm{g} / \mathrm{ml}$ of R-phycoerythrin-labeled antiCD45 (clone 30-F11), $0.20 \mu \mathrm{g} / \mathrm{ml}$ Cy-Chrome-labeled anti-CD8 (clone 53-6.7), and $0.25 \mu \mathrm{g} / \mathrm{ml}$ of FITIC-labeled anti-CD4 (clone RM 4-5) (all mAb's from Pharmingen; and R\&D Systems Inc., Minneapolis, Minnesota, USA). Washed cells were gated for $\mathrm{CD} 45^{+}$cells and size and analyzed by FACS for staining by the specific marker.

Delayed-type hypersensitivity response assay. Delayed-type hypersensitivity (DTH) response in mice was assessed by injecting purified cryptococcal antigen (CneAg) in the footpad (30). CneAg (20 $\mu \mathrm{g}$ protein $/ \mathrm{ml})$ was prepared by concentrating and dialyzing the supernatant of asparagine broth cultures of C. neoformans. Mice were first infected with SM or MC, and then at day 12 or day 23 hind footpads were injected with $30 \mu \mathrm{l} \mathrm{CneAg}$ or with uninfected asparagine broth (control). Footpad swelling was determined after 48 hours with a micrometer (Manostat Corp., New York, New York, USA) by subtracting the measurement of the control from the CneAg-injected footpad. Age-matched uninfected mice were also challenged as a negative control.

Cytokine and chemokine studies. Groups of five BALB/c mice were infected intratracheally with $10^{6}$ cells of SM, MC, or PBS only (sham). Mice were killed at days 1,3 , and 14 and the right lung was homogenized in 2 $\mathrm{ml}$ of PBS in the presence of protease inhibitors (Complete Mini; Boehringer Mannheim Biochemicals Inc., Indianapolis, Indiana, USA). The homogenate was centrifuged and the supernatant was frozen at $80^{\circ} \mathrm{C}$ until tested. The supernatants were assayed for concentrations of IL-2, IL-4, IL-6, IL-10, IL-12 p70, TNF- $\alpha$, IFN- $\gamma$, KC, monocyte chemoattractant protein-1 (MCP-1), and macrophage inflammatory protein-1 $\alpha$ (MIP-1 $\alpha$ ) using ELISA kits (Pharmingen and $\mathrm{R} \& \mathrm{D}$ Systems Inc.). The detection limits of these assays: were $3.1 \mathrm{pg} / \mathrm{ml}$ for IL-2, $7.8 \mathrm{pg} / \mathrm{ml}$ for IL-4, $31.25 \mathrm{pg} / \mathrm{ml}$ for IL-10 and IFN- $\gamma, 15.6 \mathrm{pg} / \mathrm{ml}$ for IL-6 and TNF- $\alpha, 62.5 \mathrm{pg} / \mathrm{ml}$ for IL- $12 \mathrm{p} 40,1.5 \mathrm{pg} / \mathrm{ml}$ for MIP- $1 \alpha, 15.6 \mathrm{pg} / \mathrm{ml}$ for MCP-1, and $2.0 \mathrm{pg} / \mathrm{ml}$ for KC.

Statistical analysis and Poisson probability. The Poisson formula describes the probability of the occurrence of rare events $(k): P(k)=\mathrm{e}^{-\mathrm{i}} \mathrm{i}^{k} / k !$. $P$ is the probability to observe $k$ switching events, if the mean value for the switching probability in the sample is ì. We calculated the probability that recovery of $\mathrm{MC}$ colonies from mice infected with SM (SMmice) was a result of inadvertent inoculation with a mixed inoculum.

The Poisson probability to find one MC cell in an inoculum of $10^{3} \mathrm{SM}$ cells if the expected switching frequency is $5 \%$ becomes $P_{0.05}(1)=\mathrm{e}^{-0.05} \times 0.05^{1} / 1 !=0.0475$ and $P_{0.05}(0)=\mathrm{e}^{-0.05} \times 0.05^{0} / 0 !=0.951$ to find no MC cell. The probability that all nine of ten mice received an inoculum with one MC cell (because of SM-to-MC switching in vitro prior to infection event) is calculated as the product of the corresponding Poisson probabilities $P=P_{0.05}(1)^{9} \times P_{0.05}(0)^{1}$. The formula assumes that (a) the switching event is Poisson-distributed; (b) individual switching events are independent from each other; and (c) the percentage of recovered MC colonies was not taken into account, because the actual in vivo growth rate of $\mathrm{MC}$ versus $\mathrm{SM}$ is not known.

\section{Results}

Characterization of the C. neoformans strain RC-2 as a switching system. Strain RC-2 grows as a SM colony on SDA with mucoid MC colonies arising spontaneously at a frequency of 0.5 in $10^{-4}$ (Figure 1a). Both are common colony phenotypes in clinical isolates. The reversion of MC to SM occurs in vitro at a compara-

\section{Table 1}

Characteristics of SM and MC phenotypes

\begin{tabular}{lcc}
\hline & $\begin{array}{c}\text { RC-2 SM } \\
\text { colony type }\end{array}$ & $\begin{array}{c}\text { RC-2 MC } \\
\text { colony type }\end{array}$ \\
ubling time at $30^{\circ} \mathrm{C}$ & $2.5 \mathrm{~h}$ & $2.8 \mathrm{~h}$ \\
size at $37^{\circ} \mathrm{C}$ & $2.6 \mathrm{~h}$ & $2.7 \mathrm{~h}$ \\
sule size at $37^{\circ} \mathrm{C}$ & $5.9 \pm 0.3$ & $7.1 \pm 0.79$ \\
sule size in $5 \% \mathrm{CO}_{2}$ & $1.7 \pm 0.6$ & $2.9 \pm 0.54$ \\
anization & $6.2 \pm 0.09$ & $6.9 \pm 0.05$ \\
Yes & Yes \\
M triad structure & $\mathrm{M} 1$ & $\mathrm{M} 1$
\end{tabular}

as determined by NMR

Elementary composition $\left(\mathrm{C}, \mathrm{O}_{2}, \mathrm{H}\right.$, and $\left.\mathrm{N}\right)$

Viscosity of GXM

No difference

Cell charge

$\mathrm{MC} \gg>\mathrm{SM}$

$27.68 \pm 2.44$

$30.28 \pm 3.6$ 


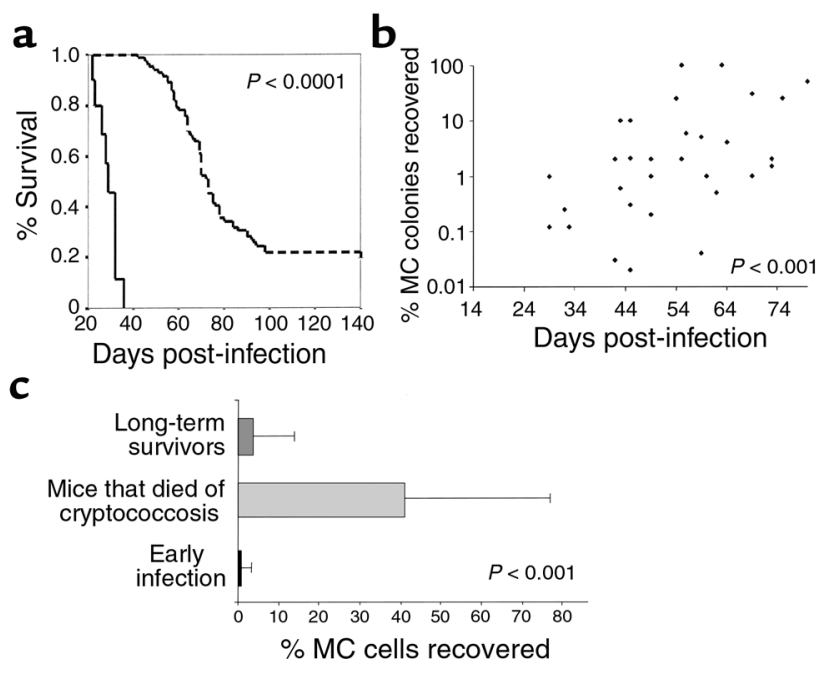

ble frequency. Karyotype analysis and RFLP patterns with an $\alpha$ - $\mathrm{P}^{32}$-labeled probe to repetitive sequences in the C. neoformans genome (CNRE) (27) did not detect DNA rearrangements and confirmed that SM and $\mathrm{MC}$ are one strain (data not shown). Comparison of several virulence traits including doubling time and the ability to melanize revealed no major differences between SM and MC (Table 1). Small differences in cell and capsule size were observed, but cell charge and sugar assimilation profile were not affected by phenotypic switching (data not shown).

Analysis of the SM and MC GXM repeat structure by NMR revealed that both GXMs were composed of the M1 structural reporter group, as expected for serotype

\section{Figure 2}

Association of phenotypic switching with virulence. (a) Kaplan Meyer survival curve shows decreased survival of mice infected intratracheally with $10^{4} \mathrm{MC}$ compared with SM. (b) Mice that are infected for a prolonged time have a higher percentage of $\mathrm{MC}$ colonies ( $P$ value, Spearman coefficient). (c) Mice that died of cryptococcosis have a higher percentage of $\mathrm{MC}$ colonies than do long-term survivors $(P=0.0001$, $t$ test) and mice prior to onset of disease $(P<0.0001, t$ test $)$. Comparison of all groups by ANOVA analysis is $P<0.001$.

D (13). However, MC GXM was more viscous than SM GXM, suggesting a difference in molecular weight (Table 1). Analysis of the molecular weight of SM and MC GXM by two independent methods, size exclusion chromatography (Figure $1 \mathrm{~b}$ ) and zonal fractionation by ultracentrifugation (Figure 1c), determined that MC GXM was larger than SM GXM.

Increased virulence of the $M C$ phenotype in mice. $\mathrm{MC}$ was more virulent than SM in BALB/c and $\mathrm{A} / \mathrm{JCr}$ mice (Figure 2a and Table 2). Mice infected with MC (MCmice) died 24 to more than 52 days earlier than SMmice. MC colonies derived from lung and CNS after in vivo switching or from in vitro switching events exhibited similar virulence. Mixed infection experiments using a 1:1 mixture of SM and MC revealed survival comparable to that of mice infected with $\mathrm{MC}$ alone, suggesting that cross-protection could not be achieved. These results demonstrate that phenotypic switching from SM to MC results in a more virulent phenotype.

Pilot experiments showing that both SM and MC colonies could be recovered from SMmice suggested either that phenotypic switching from SM to MC

Table 2

Survival difference of $\mathrm{A} / \mathrm{JCr}$ and $\mathrm{BALB} / \mathrm{c}$ mice infected with $\mathrm{SM}$ and $\mathrm{MC}^{\mathrm{A}}$

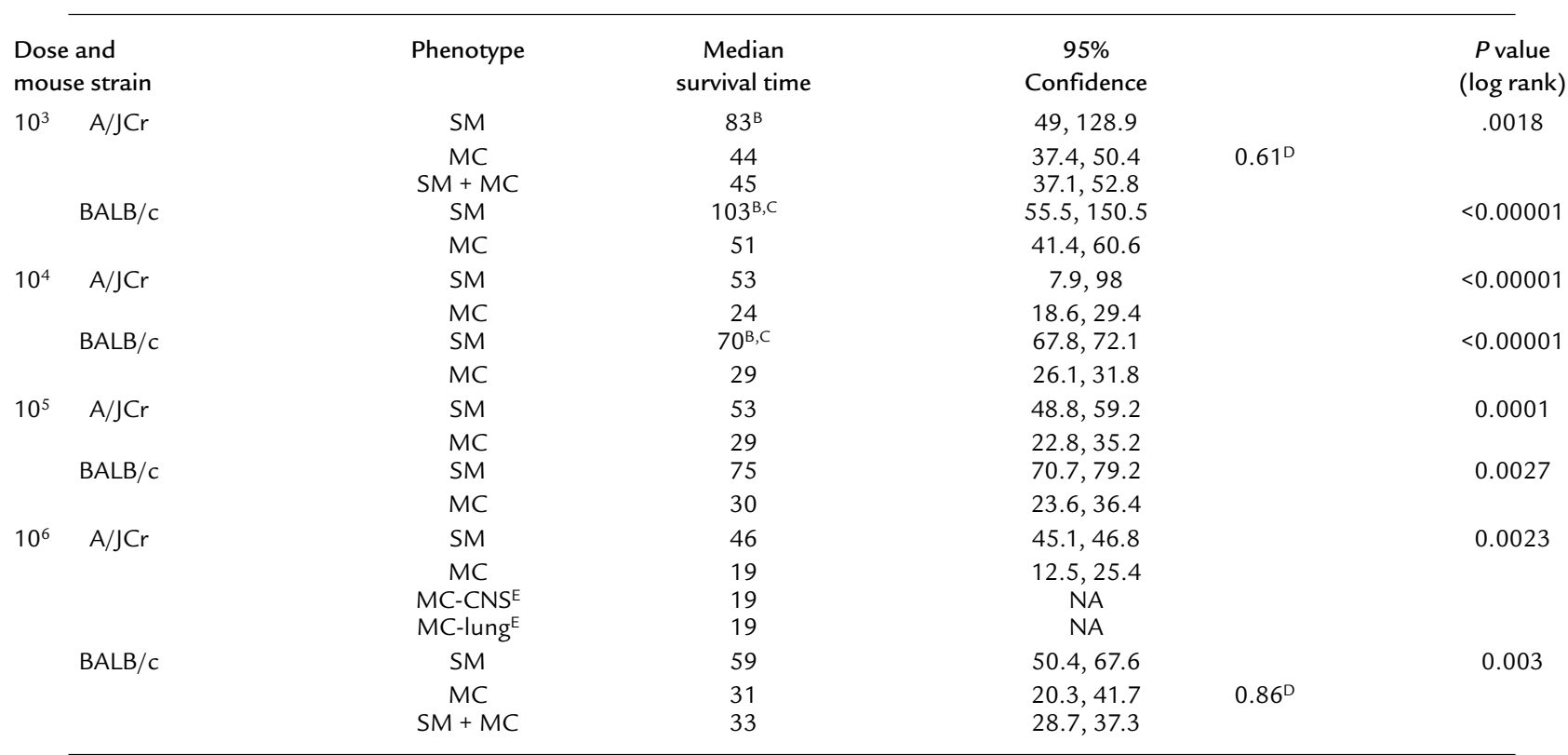

${ }^{A} P$ values were calculated by log rank regression analysis. ${ }^{B}$ Mice killed for CFU (censored). C ${ }^{C}$ ong-term survivors (more than 140 days). ${ }^{D}$ Mice received $1: 1$ mixture of SM and MC ( $P$ value compares survival with that of mice injected with MC only). EMC colonies derived from lung and CNS after in vivo switching. 


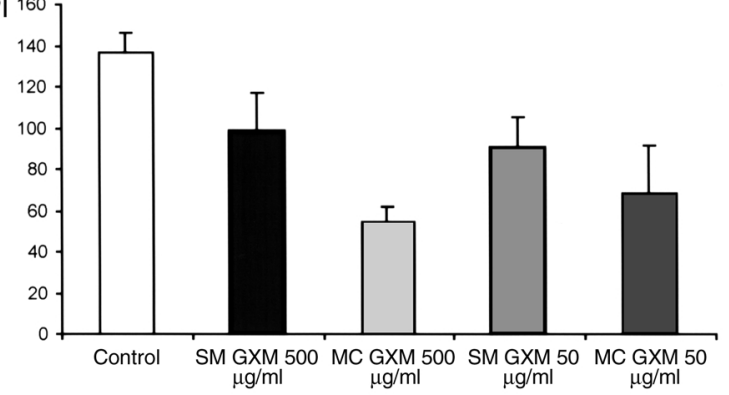

b

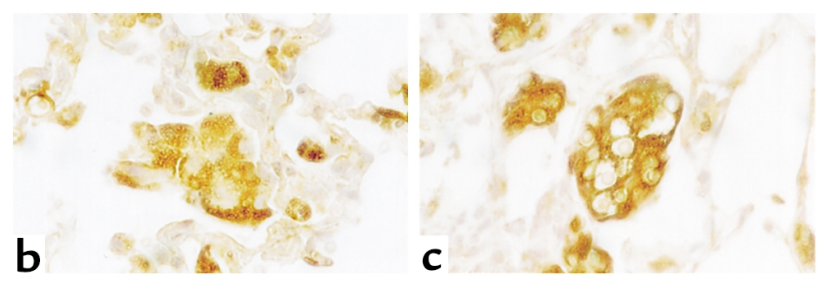

Figure 3

Effects of SM and MC GXM on phagocytosis in vitro and in vivo. (a) Inhibition of phagocytosis of Cap67mutant cells by SM and MC GXM. Phagocytosis was significantly more inhibited (63\% vs. $23 \%$, $P=0.003, t$ test) by $500 \mu \mathrm{g}$ MC GXM than SM GXM. Control is PI of Cap67 cells without GXM. (b) Formation of vacuoles in M $\phi$ 's of SMmice are the result of digested C. neoformans. (c) In contrast, intracellular persistence of C. neoformans is observed in M $\phi$ 's of MCmice.

occurred in vivo, or that rare switching events had occurred in vitro prior to infection and were selected during the infection process. To discriminate between these possibilities, the inoculum was reduced to $10^{3}$ to decrease the probability of injecting a cocktail containing at least one $\mathrm{MC}$ cell to $5 \%$. Groups of $10 \mathrm{~A} / \mathrm{JCr}$ and $11 \mathrm{BALB} / \mathrm{c}$ mice were injected with $10^{3} \mathrm{SM}$ cells, and their organs were assayed for $\mathrm{MC}$ colonies at day 45, day 73 (after infection), and the time of death. MC colonies were recovered from 9 of $10 \mathrm{~A} / \mathrm{JCr}$ and 7 of 11 $\mathrm{BALB} / \mathrm{c}$ mice (range $0.1-30 \% \mathrm{MC}$ colonies per mouse) after infection. At day 45 no MC colonies were recovered from four $\mathrm{BALB} / \mathrm{c}$ mice and one $\mathrm{A} / \mathrm{JCr}$ mouse. Application of the Poisson formula revealed that the probability of recovering $\mathrm{MC}$ colonies because of inadvertent inoculation of a MC cell was exceedingly low $\left(P=1.1 \times 10^{-12}\right.$ for $\mathrm{A} / \mathrm{JCr}$ and $P=4.4 \times 10^{-8}$ for $\mathrm{BALB} / \mathrm{c})$. Hence, the data imply that the occurrence of $\mathrm{MC}$ colonies in SMmice is the result of in vivo phenotypic switching.

To ascertain whether an association existed between phenotypic switching, duration of infection, and/or fungal organ burden (CFU), BALB/c and $\mathrm{A} / \mathrm{JCr}$ mice $(n=101)$ were infected with SM $\left(10^{3}\right.$ to $\left.10^{6}\right)$ and analyzed for the percentage of MC colonies and CFU at different times after infection. Pearson correlation analysis revealed a time-dependent increase in the percentage of MC colonies recovered from mouse organs $(P<0.001)$ (Figure $2 \mathrm{~b})$, but no correlation with CFU (data not shown). The percentage of MC colonies ranged from $0.1 \%$ to $100 \%$ in mice infected with the same dose, and analyzed at the same time. In some cases $\mathrm{MC}$ colonies were recovered only from lung or brain, indicating that phenotypic switching occurred after dissemination from lung to brain.

We then investigated the impact of phenotypic switching on the outcome of infection. BALB/c mice $(n=108)$ were infected with $10^{4} \mathrm{SM}$ cells, an inoculum that is generally cleared within 3 months by BALB/c mice. Mice autopsied before the onset of disease (before day 36) and long-term survivors had significantly $(P<0.001$, ANOVA) fewer MC colonies $(1.0 \% \pm 2.2 \%$ and $3.6 \% \pm 10.2 \%)$ than those $(41 \% \pm 35 \%)$ autopsied at the time of death between day 42 and day 140 (Figure 2c). These data demonstrate that the occurrence of phenotypic switching in vivo increases the likelihood of death following C. neoformans infection.

Mechanisms of enhanced virulence of the MC phenotype: $M C$ cells resist phagocytosis. Given the difference in GXM molecular weight and virulence, we examined the ability of different $\mathrm{M} \phi$ types to phagocytose and support intracellular replication of SM and MC. Phagocytosis of MC was significantly reduced compared with SM cells both in vitro and in vivo (Table 3). Next, we investigated whether the MC and SM GXMs differed in their ability to inhibit phagocytosis. Both SM and MC GXM significantly inhibited phagocytosis of acapsular C. neoformans cells (Cap67) mutant cells ( $P=0.001$, ANOVA). However, the addition of $500 \mu \mathrm{g} / \mathrm{ml} \mathrm{MC} \mathrm{GXM} 1$ hour before phagocytosis (Figure 3a) reduced the phagocytosis of Cap67 cells significantly more than SM GXM ( $63 \%$ vs. $24 \%$ for MC and SM GXM, $P=0.003, t$ test). The same effect was seen when MC GXM was added to Cap67 mutant cells at the time of phagocytosis (40\% vs. $7 \%$ for MC and SM GXM, $P=0.04$, t-test). Hence, MC GXM was a more effective inhibitor of phagocytosis.

Histological examination of SM-infected lungs revealed vacuolated alveolar M $\phi$ 's containing digested yeast cells (Figure 3b); in contrast, predominantly undigested encapsulated yeast cells were noted in alveolar M $\phi$ 's of MCmice (Figure 3c). This may reflect a difference in intracellular survival in vivo; however, we could not document this with our killing assays in vitro (data not shown).

$M C$ cells elicit a greater inflammatory response than SM cells. Consistent with survival differences, the inflam-

Table 3

Comparison of phagocytosis of SM and MC cells in different Mф's

\begin{tabular}{|c|c|c|}
\hline Cell line & $\begin{array}{l}\text { Phagocytosis } \\
\text { of SM cells }\end{array}$ & $\begin{array}{l}\text { Phagocytosis } \\
\text { of MC cells }\end{array}$ \\
\hline
\end{tabular}

In vitro

$\mathrm{J} 774$

$\mathrm{MH}-\mathrm{S}^{\mathrm{A}}$ $192 \pm 17$

Peritoneal M $\phi$ 's

$17.5 \pm 0.7$

Alveolar M $\phi$ 's

$74.1 \pm 0.7$

$39.16 \pm 8.6$

$74 \pm 9.4$
$12.1 \pm 0.7$
$37.9 \pm 7$
$8.9 \pm 2.0$

$P=0.02$

In vivo

Alveolar M $\phi^{\prime} \mathrm{s}^{\mathrm{B}}$

$19.9 \pm 4.2$

$3.51 \pm 1.0$

$P=0.0002$

${ }^{\mathrm{A}} \mathrm{M} \phi / \mathrm{C}$. neoformans ratio was 10:1. ${ }^{\mathrm{B}}$ Obtained from mice by bronchial alveolar lavage 4 hours after intratracheal infection. 

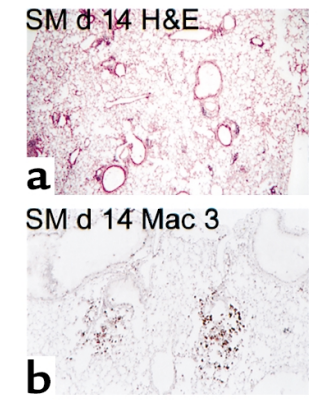

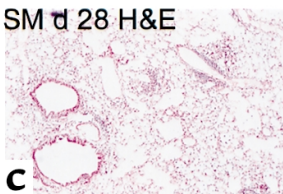

SM d 60 MUC

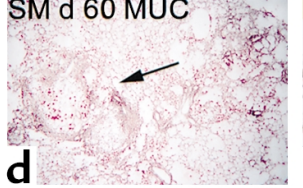

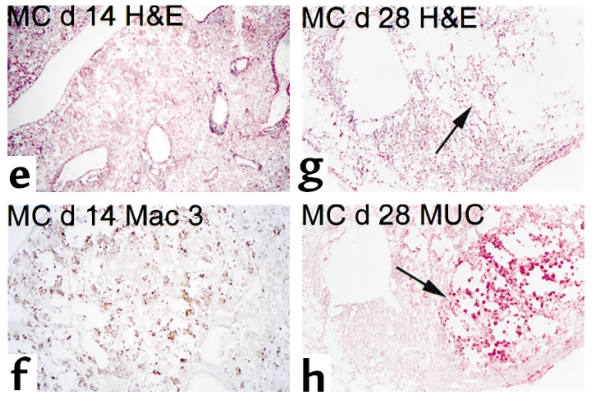

\section{Figure 4}

Inflammatory immune response in SM- and MCmice. (a-d) Histological analysis of SM-infected lungs. (a and b) At day 14, moderate inflammation (H\&E, $\times 20$; a) with few Mф's (Mac 3, $\times 20$; b). (c) At day 28, minimal destruction of lung tissue (H\&E, $\times 50)$. (d) At day 60, granuloma formation and containment of C. neoformans (arrow; Mucicarmine [MUC] stain, $\times 50)$. (e-h) In contrast, in MCmice at day 14, severe inflammation (H\&E, $\times 20$; e) composed of many Mф's (Mac 3, $\times 20 ; \mathbf{f})$. At day 28, (g) extensive destruction of alveolar membranes (arrow; $\mathrm{H} \& \mathrm{E}, \times 50$ ), and (h) persistence of C. neoformans (arrow; MUC stain, $\times 50$ ).

matory tissue response elicited by SM and MC cells differed. At day 14, lungs of SMmice exhibited moderate inflammatory changes with cellular infiltrates composed primarily of lymphocytes and only a few M $\phi$ 's (Figure 4, a and b). By day 28, the cellular infiltrates had increased within the alveolar spaces with little concomitant lung damage (Figure 4c). By day 60, granuloma formation had begun and yeast cells were surrounded by organized tissue (Figure $4 \mathrm{~d}$ ), indicating successful containment of the infection. In contrast, at day 14, MC-infected lungs exhibited extensive cellular infiltrates beyond the peribronchial regions, which were predominantly composed of M $\phi$ 's and neutrophils polymorphonuclear neutrophils (PMNs) with only a few lymphocytes (Figure 4, e and f). Near the time of death (day 28), the inflammatory response increased and resulted in extensive destruction of alveolar membranes (Figure 4g). The alveolar spaces were filled with yeast cells surrounded by large deposits of polysaccharide and M $\phi$ 's (Figure $4 \mathrm{~h}$ ). Lung weights, which reflect the magnitude of the inflammatory response, GXM deposition, and organ edema, were also higher in MCmice compared with SMmice (Table 4). Over time, the fungal organ burden increased in MCmice but decreased in SMmice (Table 4). In summary, these data show that MC induced a greater and more destructive inflammatory response than SM.
Analysis of CMI. MCmice recruited four times more cells to the lung than SMmice (Figure 5a). Analysis of infiltrating cells by morphology, FACS, and immunohistochemistry showed that SMmice recruited a higher percentage of lymphocytes and a lower percentage of PMNs and M $\phi$ 's compared with MCmice (Figure $5 \mathrm{~b}$ ). Accordingly, in lung cell suspensions we found alveolar M $\phi$ s's attached to lymphocytes in SMmice (Figure 5c) and to PMNs in MCmice (Figure 5d). Because successful clearance of $C$. neoformans infection in $\mathrm{BALB} / \mathrm{c}$ mice is dependent on a functional $\mathrm{T}$ cell response, we compared lymphocyte subpopulations in infected lungs and footpad DTH response to CneAg in SM- and MCmice. The FACS analysis of digested lung cells on day 14 and day 28 documented no difference in percentage of $\mathrm{CD}^{+}$ and $\mathrm{CD}^{+}$lymphocytes for SM- and MCmice and predominance of $\mathrm{CD}^{+}$lymphocytes (data not shown). The DTH response was greater $(P<0.001$, ANOVA $)$ in MCmice than in SMmice (Figure 5e), confirming that both SM and MC can elicit an effective immune response in the footpad.

Lung cytokine and chemokine expression differs in SM- and $M C$-infected mice. Cytokine and chemokine levels were measured by ELISA in lung homogenates from SM-, MC-, and sham-infected mice at days 1, 3, and 14. In two separate experiments, the tissue concentration of all of the cytokines and chemokines studied was significant-

Table 4

Comparison of CFU, lung weights, and GXM tissue content of infected lungs

$\begin{array}{lcc} & \text { RC-2 SM } & \text { RC-2 MC } \\ & \text { Colony type } & 5.6 \pm 0.16 \\ \log \text { CFU in A/JCr lungs, day 7 } & 5.0 \pm 0.22 & 5.9 \pm 0.66^{\mathrm{A}} \\ \log \text { CFU in A/JCr lungs, day 14 } & 4.7 \pm 0.61 & 5.8 \pm 0.13 \\ \log \text { CFU in BALB/c lungs, day 14 } & 5.8 \pm 0.13 & 7.0 \pm 0.08^{\mathrm{A}} \\ \log \text { CFU in BALB/c lungs, day 21 } & 4.6 \pm 1.5 & 1.0 \pm .36^{\mathrm{A}} \\ \text { Lung weight in BALB/c, day 21 (g) } & 0.29 \pm .009 & 879(\text { range 197-1,420) } \\ \text { GXM content in left lung, day 8 (mg) } & 653 \text { (range 321-824) } \\ \text { GXM content in left lung, day 14 (mg) } & 554 \text { (range 103-1,356) } & 1079(\text { range 51-742) }\end{array}$

AStatistically significant $P<0.05$ by $t$ test. 

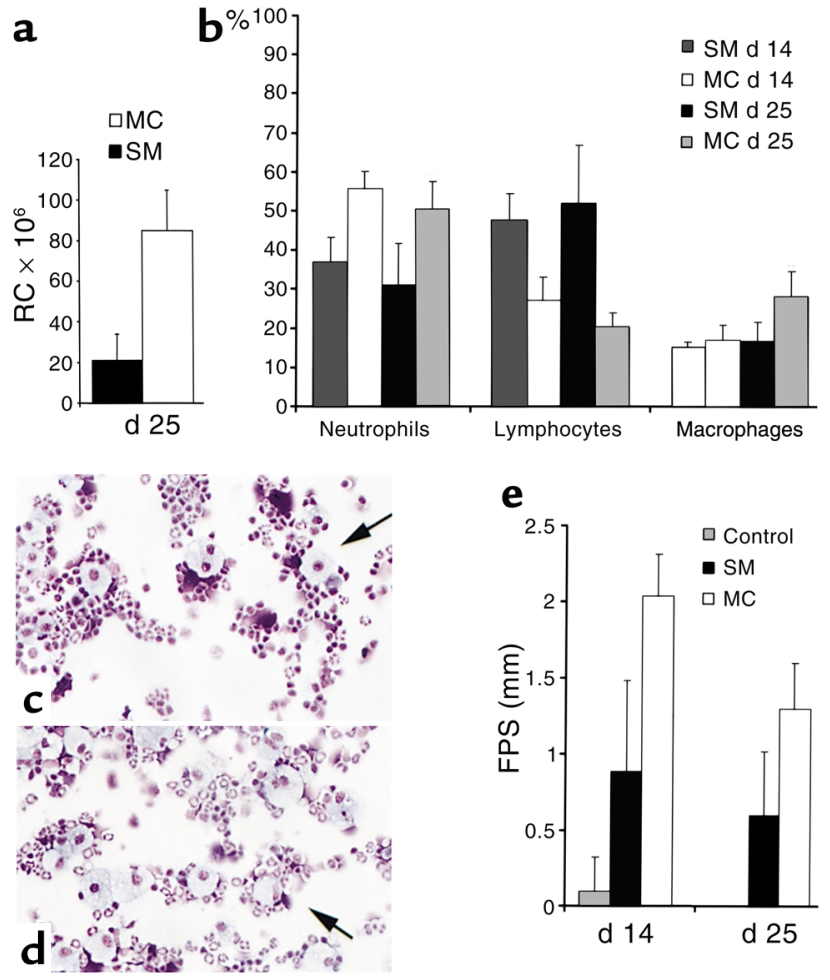

Figure 5

Analysis of inflammatory immune response. (a) Cell recruitment (RC) to lungs was four times higher in MCmice at day 25 than in SMmice $(P<0.001, t$ test $)$. (b) Morphological analysis demonstrated a higher percentage of PMNs $(P=0.016$ at day $14, P=0.001$ at day 25$)$ and M $\phi$ 's $(P=0.02$ at day 25$)$ but a lower percentage of lymphocytes ( $P=0.003$ at day 14 and $P=0.0018$ at day $25, t$ test $)$ in MCmice than in SMmice. (c and d) Stained cytospins ( $\times 20)$ of SMmice (c) showing $M \phi$ 's surrounded predominantly by lymphocytes (arrow), and of MCmice (d) surrounded predominantly by PMNs (arrow). (e) DTH testing reveals increased footpad swelling (FPS) in $\mathrm{MCmice}$ compared with SMmice at day 14 and day $25(P<0.001$, ANOVA).

ly upregulated in SM- and MC-infected compared with sham-infected mice $(P<0.02$, ANOVA). Significantly higher protein levels were detected in SM-compared with MCmice for IL-2, IL-4, IL-10, and TNF- $\alpha$ at day 14 after infection, whereas comparable tissue levels were determined for IL-6, IL-12, and IFN- $\gamma$ (Table 5). The same trend was also noted at earlier times after infection but did not reach statistical significance (data not shown). Expression of MIP- $1 \alpha$ and MCP levels was three to four times higher in MC- than in SMmice, and no difference was found for KC.

\section{Discussion}

This study demonstrates phenotypic switching for a fungus during experimental infection. The result of this switch is a MC phenotype that produces a larger GXM that reduces phagocytic efficacy by alveolar $M \phi$ 's. Our data provide strong evidence that in vivo switching enhances the virulence of $C$. neoformans by eliciting a more vigorous and qualitatively different inflammatory response that cannot effectively clear the infection but rather promotes lung tissue destruction and rapid demise.

Proof that phenotypic switching occurs in vivo has heretofore been difficult to obtain, because it is not feasible to induce an infection with a single cell. We infected mice with a small inoculum $\left(10^{3}\right)$, which was capable of causing chronic infection but unlikely to contain one MC cell (in vitro switching rate $\left[0.5 \times 10^{-4}\right]$ ) (25). Application of the Poisson formula indicated that the likelihood of MC colony recovery from SMmice as a result of mixed infection was exceedingly low $\left(P=1.1 \times 10^{-12}\right)$. Two other observations were consistent with in vivo switching: (a) mouse-to-mouse variation (ranging from $0.1 \%$ to $100 \%$ ) that would not be expected if the source of variant phenotypes were the inoculum; and (b) recovery of MC colonies exclusively from the brain in two mice, which indicates phenotypic switching in vivo after dissemination.

Our data show that a greater likelihood of death occurred in mice with evidence of in vivo switching to the $\mathrm{MC}$ phenotype (Figure 2c). Hence, an infection dose $\left(10^{4} \mathrm{CFU}\right)$ that would not be expected to be lethal for the majority of mice became more virulent by phenotypic switching $(31,32)$. Because both SM and MC are intrinsically virulent isolates, death also occurred in

\section{Table 5}

Cytokine and chemokine expression profiles at day 14 in infected lungs ${ }^{A}$

\begin{tabular}{|c|c|c|c|}
\hline $\begin{array}{l}\text { Cytokine or } \\
\text { chemokine }\end{array}$ & $\begin{array}{l}\text { Protein concentration in } \\
\text { infected lung tissue }(\mathrm{pg} / \mathrm{ml})\end{array}$ & $P$ value & \\
\hline IL-2 & $\begin{array}{l}\text { Sham } \\
\text { SM } \\
\text { MC }\end{array}$ & $\begin{array}{c}15.5 \pm 14 \\
169 \pm 125 \\
51 \pm 25\end{array}$ & 0.012 \\
\hline IL-4 & $\begin{array}{l}\text { Sham } \\
\text { SM } \\
\text { MC }\end{array}$ & $\begin{array}{c}121 \pm 78 \\
491 \pm 340 \\
219 \pm 118\end{array}$ & 0.03 \\
\hline IL-6 & $\begin{array}{l}\text { Sham } \\
\text { SM } \\
\text { MC }\end{array}$ & $\begin{array}{c}371 \pm 257 \\
981 \pm 197 \\
1,039 \pm 361\end{array}$ & No difference \\
\hline IL-10 & $\begin{array}{l}\text { Sham } \\
\text { SM } \\
\text { MC }\end{array}$ & $\begin{aligned} 479 & \pm 140 \\
2,188 & \pm 1,063 \\
861 & \pm 389\end{aligned}$ & $<0.001$ \\
\hline IL-12 & $\begin{array}{l}\text { Sham } \\
\text { SM } \\
\text { MC }\end{array}$ & $\begin{array}{c}357 \pm 157 \\
660 \pm 77 \\
533 \pm 282\end{array}$ & No difference \\
\hline IFN- $\gamma$ & $\begin{array}{l}\text { Sham } \\
\text { SM } \\
\text { MC }\end{array}$ & $\begin{array}{c}249 \pm 135 \\
1,091 \pm 218 \\
2,981 \pm 3,420\end{array}$ & No difference \\
\hline TNF- $\alpha$ & $\begin{array}{l}\text { Sham } \\
\text { SM } \\
\text { MC }\end{array}$ & $\begin{array}{c}665 \pm 265 \\
2,434 \pm 458 \\
1,333 \pm 561\end{array}$ & 0.01 \\
\hline MIP-1 $\alpha$ & $\begin{array}{l}\text { Sham } \\
\text { SM } \\
\text { MC }\end{array}$ & $\begin{array}{c}\text { Undetectable } \\
238 \pm 183 \\
1,035 \pm 443\end{array}$ & $<0.001$ \\
\hline MCP & $\begin{array}{l}\text { Sham } \\
\text { SM } \\
\text { MC }\end{array}$ & $\begin{array}{c}161 \pm 87 \\
744 \pm 724 \\
2,545 \pm 981\end{array}$ & $<0.001$ \\
\hline KC & $\begin{array}{l}\text { Sham } \\
\text { SM } \\
\text { MC }\end{array}$ & $\begin{array}{c}63 \pm 37 \\
548 \pm 300 \\
510 \pm 263\end{array}$ & No difference \\
\hline
\end{tabular}

${ }^{A} P$ values are derived from $t$ test comparing levels in $\mathrm{SM}$ - and $\mathrm{MCmice}$. Comparison of expression in all three groups (sham, SM-, and MC-infected) by ANOVA yielded $P<0.02$. 
the absence of switching. A failure of host defense that leads to an increased fungal burden is often invoked to explain enhanced cryptococcal virulence. Thus, the finding that phenotypic switching to MC augments the virulence of a C. neoformans strain is important, because it indicates that the pathogen can generate intrinsically more virulent variants in vivo.

The enhanced virulence of MC may be explained by the change in molecular weight for GXM, the predominant capsular polysaccharide and a key virulence factor. GXM is an immunomodulator (33) and can alter cytokine and chemokine production by monocytes and PMNs in vitro (34-39). GXM can be immunosuppressive in vivo (40), directly inhibit phagocytosis $(6,41)$, and delay the processing of phagocytosed C. neoformans (42). We found that phagocytosis of MC was impaired compared with SM cells (Table 3). This inhibition was more pronounced with MC than with SM GXM when phagocytosis of acapsular yeast cells was examined (Figure 3a) at GXM concentrations that were comparable to lung tissue concentrations during chronic infection (Table 4). Interestingly, previous studies using polysaccharides with variable molecular weights demonstrated that their ability to inhibit phagocytosis was a function of size, with the larger polysaccharide being more effective at inhibiting phagocytosis $(43,44)$. Whereas those studies used polysaccharides derived from different $C$. neoformans isolates, our data show that through phenotypic switching one individual C. neoformans isolate can alter the size of its polysaccharide and gain a biological advantage during infection. Unlike a hypervirulent $C$. neoformans mutant with an inactivated regulatory protein (PKR 1) of the capsule, there were no differences in capsule size under inducing conditions between the SM parent and the more virulent MC variant (Table 1) (9). Our findings are similar to those described for Pseudomonas aeruginosa, which also switches to a MC colony phenotype with an altered polysaccharide (45). Notably, the MC and more viscous polysaccharide of $C$. neoformans may create a more effective biofilm that protects it from host effector cells.

Successful phagocytosis is required for effective CMI, and proliferation of lymphocytes in response to C. neoformans correlates with the magnitude of phagocytosis $(46,47)$. Therefore, we hypothesized that impaired phagocytosis affects the host-pathogen interaction and alters the CMI. Our investigations found qualitative changes in effector cells with increased numbers of $M \phi$ 's and PMNs in MC- compared with SM-infected lungs (Figure $5 \mathrm{~b}$ ). Although other groups have reported the association of minimal inflammatory responses with a failure to clear cryptococcal infection, we have found that an enhanced inflammatory response was associated with rapid demise in MCmice $(32,48)$.

Alveolar M $\phi$ 's constitute the primary phagocytic cell in pulmonary cryptococcosis, and human autopsy studies indicate that PMNs are recruited primarily in response to tissue damage resulting from infection (49). Our findings suggest that impaired phagocytosis leads to upregulation of chemokines MCP and MIP- $1 \alpha$, which in turn leads to an increased influx of M $\phi$ 's. We propose that, analogous to other chronic inflammatory lung diseases, such as pneumoconiosis (50), overactivation of alveolar $M \phi$ 's results in the release of inflammatory mediators that promote destruction of lung tissue. In support of this concept, SMmice exhibited higher levels of IL-10, a potent downregulator of proinflammatory cytokines in vitro $(51,52)$ and in vivo $(53)$. Although a significant increase in the examined proinflammatory cytokines was not documented in this study, there was a trend toward higher IFN- $\gamma$ levels in MCmice. Taken together, the differences in cell recruitment and cytokine expression may have changed the immune response from a protective, effective immune response elicited by SM to a damage-driven inflammatory response elicited by MC.

SMmice manifested effective CMI that led to granuloma formation, whereas MCmice did not. In $\mathrm{BALB} / \mathrm{c}$ mice, successful clearance of C. neoformans infection is ultimately dependent on a Th1 response, which has been associated with the ability to mount a DTH response against CneAg $(31,32)$. Despite a failure to clear pulmonary infection, MCmice were still able to elicit a strong DTH response to CneAg in the footpad (Figure 5e). A dichotomy between relative degrees of DTH and pulmonary resistance has been reported previously (32). In this study, it most likely reflects a failure of MCmice to locally activate lymphocytes in the inflamed lungs despite an increased number of recruited cells including $\mathrm{CD}^{+}$and $\mathrm{CD}^{+}$ lymphocytes. In support of this concept, we observed differences in cytokine profiles in MC-infected lungs. Despite increased cell recruitment in MC-infected lungs, we found significantly lower levels of cytokines that are critical for the development of protective $T$ cell immunity $(54,55)$, namely, IL-2, IL-4, and TNF- $\alpha$ (Table 5). Because most of these cytokines are produced by more than one cell type, we cannot determine which cells contributed to the observed levels. Nonetheless, as antigen-presenting cells, alveolar M $\phi$ 's play a pivotal role in orchestrating CMI, and, as such, they directly or indirectly control the production of cytokines such as TNF- $\alpha$, IL-12, and IFN- $\gamma$ (49, 56-58). In this regard, C. neoformans-laden monocytes can interact with $T$ cells and promote the secretion of proinflammatory cytokines (38), and capsular polysaccharide can inhibit $\mathrm{T}$ cell proliferation by downregulating IL-2 production (59). Based on our results, we propose a model whereby altered GXM size in MC cells renders them more resistant to phagocytosis and impairs antigen presentation and lymphocyte activation. This in turn causes an upregulation of chemokines, which then increases recruitment of cells and promotes a damage-driven noneffective immune response that leads to rapid demise.

Phenotypic switching has been thoroughly studied in Candida albicans $(19,20)$ and, most recently, in Candida glabrata (21). In Candida the newly generated colony types are also associated with changes in virulence when reinoculated into animals. The switching C. albicans strain $3153 \mathrm{~A}$ and C. neoformans strain 
24067a exhibit an association, but no clear correlation, between karyotype changes and switching $(23,60)$. The variant RC-2 described here has a stable chromosome length change of one chromosome that differs from the original 24067 pattern (13) but does not manifest karyotype changes with switching. Similarly, no changes were detected in RFLP patterns of genomic DNA hybridized with CNRE when SM and MC variants were compared. Although the mechanism(s) responsible for switching in C. neoformans is not known, this process may occur without DNA rearrangement. In Candida, epigenetic mechanisms such as SIR2-mediated silencing and HDA1-mediated acetylation of histones play a role in regulating switching frequencies $(60,61)$.

In summary, $C$. neoformans provides an excellent model system to study the contribution of phenotypic switching to virulence, because chronic infections can be established by innoculation with a relatively low number of C. neoformans cells. This allowed us to demonstrate that phenotypic switching occurs in vivo, and that it affects the outcome of infection. Enhanced virulence in our model was the result of a dysfunctional immune response to an altered polysaccharide capsule as a consequence of phenotypic switching. The importance of the occurrence of phenotypic switching in vivo is that it regulates key virulence factors and leaves the host at risk for progression without the change of host immunity.

\section{Acknowledgments}

We thank D. Fries for help with the Poisson analysis, A. Rosas for assistance with phagocytosis assays, J. Nosanchuk for measuring cell charge, and L. Pirofski for critical review of the manuscript. This work was supported in part by NIH (RO-1-AI33774, AI3342, HL59842 to A. Casadevall, and KO8-AI01570 to B.C. Fries), Fundação de Amparo à Pesquisa do Estado de São Paulo (FAPESP 99/00185-1 to C.P. Taborda), and Howard Hughes Medical Institute (postdoctoral fellowship to B.C. Fries).

1. Mitchell, T., and Perfect, J. 1995. Cryptococcosis in the era of AIDS: 100 years after the discovery of Cryptococcus neoformans. Clin. Microbiol. Rev. 8:515-548.

2. Kwon-Chung, K., Polacheck, J., and Popkin, T. 1982. Melanin lacking mutants of Cryptococcus neoformans and their virulence for mice. J. Bacteriol. 150:1414-1421.

3. Kwon-Chung, K.J., Edman, J.C., and Wickes, B.L. 1992. Genetic association of mating types and virulence in Cryptococcus neoformans. Infect. Immun. 60:602-605

4. Cox, G.M., et al. 2001. Extracellular phospholipase activity is a virulence factor for Cryptococcus neoformans. Mol. Microbiol. 39:166-175.

5. Odom, A., et al. 1997. Calcineurin is required for virulence of Cryptococcus neoformans. EMBOJ. 16:2576-2589.

6. Bulmer, G.S., and Sans, M.D. 1968. Cryptococcus neoformans. III. Inhibition of phagocytosis. J. Bacteriol. 95:5-8.

7. Granger, D.L., Perfect, J.R., and Durack, D.T. 1985. Virulence of Cryptococcus neoformans. Regulation of capsule synthesis by carbon dioxide. J. Clin. Invest. 76:508-516.

8. Perfect, J.R. 1996. Fungal virulence genes as targets for antifungal chemotherapy. Antimicrob. Agents Chemother. 40:1577-1583.

9. D'Souza, C.A., et al. 2001. Cyclic AMP-dependent protein kinase controls virulence of the fungal pathogen Cryptococcus neoformans. Mol. Cell. Biol. 21:3179-3191.

10. Alspaugh, J.A., Perfect, J.R., and Heitman, J. 1997. Cryptococcus neoformans mating and virulence are regulated by the G- protein alpha subunit GPA1 and cAMP. Genes Dev. 11:3206-3217.

11. Cherniak, R., Morris, L., Belay, T., Spitzer, E., and Casadevall, A. 1995. Variation in the structure of glucuronoxylomannan in isolates from patients with recurrent Cryptococcal meningitis. Infect. Immun. 63:1899-1905.

12. Currie, B., et al. 1995. Sterol compositions and susceptibilities to amphotericin B of environmental Cryptococcus neoformans isolates are changed by murine passage. Antimicrob. Agents Chemother. 39:1934-1937.

13. Franzot, S.P., et al. 1998. Microevolution of a standard strain of Cryptococcus neoformans resulting in differences in virulence and other phenotypes. Infect. Immun. 66:89-97.

14. Chen, L.C., and Casadevall, A. 1999. Variants of a Cryptococcus neoformans strain elicit different inflammatory responses in mice. Clin. Diagn. Lab. Immunol. 6:266-268.

15. Fries, B.C., and Casadevall, A. 1998. Serial isolates of Cryptococcus neoformans from patients with AIDS differ in virulence for mice. J. Infect. Dis. 178:1761-1766.

16. Speed, B., and Dunt, D. 1995. Clinical and host differences between infections with the two varieties of Cryptococcus neoformans. Clin. Infect. Dis. 21:28-34.

17. Reznick, D.N., Shaw, F.H., Rodd, F.H., and Shaw, R.G. 1997. Evaluation of the rate of evolution in natural populations of guppies (Poecilia reticulata). Science. 275:1934-1937.

18. Deitsch, K.W., Moxon, E.R., and Wellems, T.E. 1997. Shared themes of antigenic variation and virulence in bacterial, protozoal, and fungal infections. Microbiol. Mol. Biol. Rev. 61:281-293.

19. Slutsky, B., et al. 1987. "White-opaque transition": a second high-frequency switching system in Candida albicans. J. Bacteriol. 169:189-197.

20. Slutsky, B., Buffo, J., and Soll, D.R. 1985. High-frequency switching of colony morphology in Candida albicans. Science. 230:666-669.

21. Lachke, S.A., Srikantha, T., Tsai, L.K., Daniels, K., and Soll, D.R. 2000. Phenotypic switching in Candida glabrata involves phase-specific regulation of the metallothionein gene MT-II and the newly discovered hemolysin gene HLP. Infect. Immun. 68:884-895.

22. Goldman, D.L., Fries, B.C., Franzot, S.P., Montella, L., and Casadevall, A. 1998. Phenotypic switching in the human pathogenic fungus Cryptococcus neoformans is associated with changes in virulence and pulmonary inflammatory response in rodents. Proc. Natl. Acad. Sci. USA. 95:14967-14972.

23. Fries, B.C., Goldman, D.L., Cherniak, R., Ju, R., and Casadevall, A. 1999. Phenotypic switching in Cryptococcus neoformans results in changes in cellular morphology and glucuronoxylomannan structure. Infect. Immun. 67:6076-6083.

24. Schwan, T.G., and Hinnebush, B.J. 1998. Bloodstream- versus tick-associated variants of a relapsing fever bacterium. Science. 280:1938-1940.

25. Ebert, D. 1998. Experimental evolution of parasites. Science. 282:1432-1435.

26. Nosanchuk, J.D., and Casadevall, A. 1997. Cellular charge of Cryptococcus neoformans: contributions from the capsular polysaccharide, melanin, and monoclonal antibody binding. Infect. Immun. 65:1836-1841.

27. Spitzer, E.D., and Spitzer, S.G. 1992. Use of a dispersed repetitive DNA element to distinguish clinical isolates of Cryptococcus neoformans. J. Clin. Microbiol. 30:1094-1097.

28. Taborda, C.P., and Casadevall, A. 2001. Immunoglobulin M efficacy against Cryptococcus neoformans: mechanism, dose dependence, and prozone-like effects in passive protection experiments. J. Immunol. 166:2100-2107.

29. Huffnagle, G.B., et al. 2000. Leukocyte recruitment during pulmonary Cryptococcus neoformans infection. Immunopharmacology. 48:231-236.

30. Murphy, J.W., Schafer, F., Casadevall, A., and Adesina, A. 1998. Antigeninduced protective and nonprotective cell-mediated immune components against Cryptococcus neoformans. Infect. Immun. 66:2632-2639.

31. Huffnagle, G.B., Yates, J.L., and Lipscomb, M.F. 1991. Immunity to a pulmonary Cryptococcus neoformans infection requires both CD4+ and CD8+ T cells. J. Exp. Med. 173:793-800.

32. Huffnagle, G.B., Yates, J.L., and Lipscomb, M.F. 1991. T cell-mediated immunity in the lung: a Cryptococcus neoformans pulmonary infection model using SCID and athymic nude mice. Infect. Immun. 59:1423-1433.

33. Vecchiarelli, A. 2000. Immunoregulation by capsular components of Cryptococcus neoformans. Med. Mycol. 38:407-417.

34. Vecchiarelli, A., et al. 1995. Downregulation by cryptococcal polysaccharide of tumor necrosis factor alpha and interleukin-1 beta secretion from human monocytes. Infect. Immun. 63:2919-2923.

35. Retini, C., et al. 1996. Capsular polysaccharide of Cryptococcus neoformans induces proinflammatory cytokine release by human neutrophils. Infect. Immun. 64:2897-2903.

36. Vecchiarelli, A., et al. 1996. Purified capsular polysaccharide of Cryptococcus neoformans induces interleukin- 10 secretion by human monocytes. Infect. Immun. 64:2846-2849.

37. Lendvai, N., Qu, X.W., Hsueh, W., and Casadevall, A. 2000. Mechanism for the isotype dependence of antibody-mediated toxicity in Cryptococcus 
neoformans-infected mice. J. Immunol. 164:4367-4374.

38. Vecchiarelli, A., Retini, C., Pietrella, D., Monari, C., and Kozel, T.R. 2000 $\mathrm{T}$ lymphocyte and monocyte interaction by CD40/CD40 ligand facilitates a lymphoproliferative response and killing of Cryptococcus neoformans in vitro. Eur. J. Immunol. 30:1385-1393.

39. Mozaffarian, N., Casadevall, A., and Berman, J.W. 2000. Inhibition of human endothelial cell chemokine production by the opportunistic fungal pathogen Cryptococcus neoformans. J. Immunol. 165:1541-1547.

40. Mody, C.H., and Syme, R.M. 1993. Effect of polysaccharide capsule and methods of preparation on human lymphocyte proliferation in response to Cryptococcus neoformans. Infect. Immun. 61:464-469.

41. Kozel, T.R., and Mastroianni, R.P. 1976. Inhibition of phagocytosis by cryptococcal polysaccharide: dissociation of the attachment and ingestion phases of phagocytosis. Infect. Immun. 14:62-67.

42. Collins, H., and Bancroft, G. 1991. Encapsulation of Cryptococcus neofor mans impairs antigen-specific $\mathrm{T}$ cell responses. Infect. Immun. 59:3883-3886

43. Small, J., and Mitchell, T. 1989. Strain variation in antiphagocytic activity of capsular polysaccharide from Cryptococcus neoformans serotype A. Infect. Immun. 57:3751-3756.

44. Yasuoka, A., Kohno, S., Yamada, H., Kaku, M., and Koga, H. 1994. Influence of molecular sizes of Cryptococcus neoformans capsular polysaccharide on phagocytosis. Microbiol. Immunol. 38:851-856.

45. Mathee, K., et al. 1999. Mucoid conversion of Pseudomonas aeruginosa by hydrogen peroxide: a mechanism for virulence activation in the cystic fibrosis lung. Microbiology. 145:1349-1357.

46. Syme, R.M., Bruno, T.F., Kozel, T.R., and Mody, C.H. 1999. The capsule of Cryptococcus neoformans reduces T-lymphocyte proliferation by reducing phagocytosis, which can be restored with anticapsular antibody. Infect. Immun. 67:4620-4627.

47. Syme, R.M., Spurrell, J.C., Ma, L.L., Green, F.H., and Mody, C.H. 2000 Phagocytosis and protein processing are required for presentation of Cryptococcus neoformans mitogen to $\mathrm{T}$ lymphocytes. Infect. Immun. 68:6147-6153

48. Blackstock, R., Buchanan, K.L., Adesina, A.M., and Murphy, J.W. 1999. Differential regulation of immune responses by highly and weakly virulent Cryptococcus neoformans isolates. Infect. Immun. 67:3601-3609.

49. Feldmesser, M., Kress, Y., Novikoff, P., and Casadevall, A. 2000. Crypto- coccus neoformans is a facultative intracellular pathogen in murine pulmonary infection. Infect. Immun. 68:4225-4237.

50. Vanhee, D., Gosset, P., Boitelle, A., Wallaert, B., and Tonnel, A.B. 1995 Cytokines and cytokine network in silicosis and coal workers' pneumoconiosis. Eur. Respir. J. 8:834-842.

51. de Waal Malefyt, R., Abrams, J., Bennett, B., Figdor, C.G., and de Vries, J.E. 1991. Interleukin 10 (IL-10) inhibits cytokine synthesis by human monocytes: an autoregulatory role of IL-10 produced by monocytes. J. Exp. Med. 174:1209-1220.

52. Levitz, S.M., Tabuni, A., Nong, S.H., and Golenbock, D.T. 1996. Effects of interleukin-10 on human peripheral blood mononuclear cell responses to Cryptococcus neoformans, Candida albicans, and lipopolysaccharide. Infect. Immun. 64:945-951.

53. Huaux, F., et al. 1998. Role of interleukin-10 in the lung response to silica in mice. Am. J. Respir. Cell Mol. Biol. 18:51-59.

54. Huffnagle, G.B., et al. 1996. Afferent phase production of TNF-alpha is required for the development of protective $\mathrm{T}$ cell immunity to Cryptococ cus neoformans. J. Immunol. 157:4529-4536.

55. Buchanan, K.L., and Murphy, J.W. 1993. Characterization of cellular infiltrates and cytokine production during the expression phase of the anticryptococcal delayed-type hypersensitivity response. Infect. Immun. 61:2854-2865.

56. Vecchiarelli, A., et al. 1994. Role of human alveolar macrophages as antigen-presenting cells in Cryptococcus neoformans infection. Am. J. Respir. Cell Mol. Biol. 11:130-137.

57. Levitz, S.M. 1994. Macrophage-Cryptococcus interactions. Immunol. Ser. 60:533-543.

58. Levitz, S.M., et al. 1997. Variables affecting production of monocyte chemotactic factor 1 from human leukocytes stimulated with Cryptococcus neoformans. Infect. Immun. 65:903-908.

59. Vecchiarelli, A., et al. 1994. Encapsulation of Cryptococcus neoformans regulates fungicidal activity and the antigen presentation process in human alveolar macrophages. Clin. Exp. Immunol. 98:217-223.

60. Perez-Martin, J., Uria, J.A., and Johnson, AD. 1999. Phenotypic switching in Candida albicans is controlled by SIR2 gene. EMBOJ.18:2580-2592.

61. Klar, A.J., Srikantha, T., and Soll, D.R. 2001. A histone deacetylation inhibitor and mutant promote colony-type switching of the human pathogen Candida albicans. Genetics. 158:919-924. 